\title{
The gonococcal genetic island and type IV secretion in the pathogenic Neisseria
}

\section{Meghan E. Ramsey, Katelynn L. Woodhams and Joseph P. Dillard*}

Department of Medical Microbiology and Immunology, University of Wisconsin-Madison, Madison, WI, USA

\section{Edited by:}

Cynthia N. Cornelissen, Virginia Commonwealth University School of

Medicine, USA

\section{Reviewed by:}

Eric P. Skaar, Vanderbilt University, USA Nathan John Weyand, BIO5 Institute, USA

\section{*Correspondence:}

Joseph P. Dillard, Department of

Medical Microbiology and Immunology, University of Wisconsin-Madison, 6245

Microbial Sciences Building, 1550 Linden Drive, Madison, WI 53706, USA.

e-mail: jpdillard@wisc.edu
Eighty percent of Neisseria gonorrhoeae strains and some Neisseria meningitidis strains encode a 57-kb gonococcal genetic island (GGI). The GGI was horizontally acquired and is inserted in the chromosome at the replication terminus. The GGI is flanked by direct repeats, and sitespecific recombination at these sites results in excision of the $\mathrm{GGI}$ and may be responsible for its original acquisition. Although the role of the GGI in N. meningitidis is unclear, the GGI in N. gonorrhoeae encodes a type IV secretion system (T4SS). T4SS are versatile multi-protein complexes and include both conjugation systems as well as effector systems that translocate either proteins or DNA-protein complexes. In N. gonorrhoeae, theT4SS secretes single-stranded chromosomal DNA into the extracellular milieu in a contact-independent manner. Importantly, the DNA secreted through the T4SS is effective in natural transformation and therefore contributes to the spread of genetic information through Neisseria populations. Mutagenesis experiments have identified genes for DNA secretion including those encoding putative structural components of the apparatus, peptidoglycanases which may act in assembly, and relaxosome components for processing the DNA and delivering it to the apparatus. The T4SS may also play a role in infection by $N$. gonorrhoeae. During intracellular infection, $N$. gonorrhoeae requires the Ton complex for iron acquisition and survival. However, N. gonorrhoeae strains that do not express the Ton complex can survive intracellularly if they express structural components of theT4SS. These data provide evidence that the T4SS is expressed during intracellular infection and suggest that the T4SS may provide an advantage for intracellular survival. Here we review our current understanding of how the GGI and type IV secretion affect natural transformation and pathogenesis in N. gonorrhoeae and N. meningitidis.

Keywords: type IV secretion system, gonococcal genetic island, Neisseria gonorrhoeae, Neisseria meningitidis, natural transformation

\section{INTRODUCTION}

Neisseria gonorrhoeae (gonococcus, GC) and Neisseria meningitidis (meningococcus, MC) are both exclusively pathogenic to humans, causing the sexually transmitted disease gonorrhea and bacterial meningitis respectively. Approximately $80 \%$ of gonococcal strains carry a genomic island called the gonococcal genetic island (GGI; Dillard and Seifert, 2001). Recently, this island was also identified in some strains of $N$. meningitidis, although it has not been found in any of the commensal Neisseria species (Dillard and Seifert, 2001; Snyder et al., 2005). In N. gonorrhoeae, the GGI is $57 \mathrm{~kb}$ in size and is integrated into the gonococcal chromosome at the replication terminus (Dillard and Seifert, 2001; Hamilton et al., 2005).

Sequence characteristics of the GGI indicate that, like many other genomic islands in bacteria, the GGI was horizontally acquired (Hacker and Kaper, 2000; Hamilton et al., 2005). The $\mathrm{G}+\mathrm{C}$ content of the GGI is $44 \%$, significantly lower than the $51 \%$ average for the sequenced $N$. gonorrhoeae genomes. Dinucleotide frequencies also vary between the GGI and the core genome (Karlin, 1998; Hamilton et al., 2005). Additionally, horizontally acquired genetic islands are often flanked by short direct repeats (Hacker and Kaper, 2000). The GGI is flanked by copies of the dif site, and sitespecific recombination at these sites may have resulted in the initial acquisition of the island (Hamilton et al., 2005; Domínguez et al., 2011). The GGI also differs from the core genome in the number of DNA uptake sequences (DUS) it contains. The DUS is required for DNA uptake during natural transformation and is present at an average frequency of one DUS per every $1.1 \mathrm{~kb}$ in neisserial genomes (Goodman and Scocca, 1988; Smith et al., 1999). In N. gonorrhoeae strain MS11, the GGI contains only six copies of the DUS, while the $57-\mathrm{kb}$ region flanking the GGI contains 53 copies (Hamilton et al., 2005). Together, these observations suggest that the GGI was likely horizontally acquired and has stably integrated into the gonococcal chromosome.

Genetic islands often encode genes important for pathogenesis, metabolism, or ecological fitness and therefore may provide their host species with selective advantages (Hacker and Kaper, 2000; Gal-Mor and Finlay, 2006). The role of the GGI in meningococci remains unclear, but in gonococci, it has been shown that the GGI encodes a type IV secretion system (T4SS) that secretes DNA into the extracellular environment (Dillard and Seifert, 2001; Hamilton et al., 2005). Both N. gonorrhoeae and N. meningitidis are naturally competent, and the DNA secreted by the T4SS is effective for natural transformation in gonococci (Dillard and Seifert, 2001; Hamilton and Dillard, 2006). Therefore, the GGI-encoded T4SS contributes to horizontal gene transfer within Neisseria populations. 
The role of the GGI in gonococcal pathogenesis is less clear, and several studies have investigated whether the presence of the GGI is correlated with different disease types. The mere presence of the GGI does not correlate with any particular disease presentation. The GGI was identified at a similar frequency in gonococcal strains isolated from women with either symptomatic or asymptomatic infection (Wu et al., 2011). The GGI is also present at similar frequencies in low-passage clinical isolates causing both pelvic inflammatory disease and local infection. However, the GGI is variable at the traG/ atlA locus, and certain versions of the GGI have been correlated with disseminated gonococcal infections (DGI; Dillard and Seifert, 2001). TraG is a predicted T4SS protein, while AtlA is one of two peptidoglycanases encoded by the genetic island (Dillard and Seifert, 2001; Hamilton et al., 2001, 2005; Lawley et al., 2003; Kohler et al., 2007). Early work identified an allele of $\operatorname{tra} G$ that included the sac-4 locus, a locus that had previously been implicated in serum-resistance (McShan et al., 1987; Nowicki et al., 1997; Dillard and Seifert, 2001). The sac-4 allele of $\operatorname{traG}$ is not present in all GGI variants, however, and the presence of the atlA gene is also variable (Dillard and Seifert, 2001). In a survey of low-passage clinical isolates, atlA and the sac-4 allele of traG were each correlated with isolates causing DGI (Dillard and Seifert, 2001). These observations suggest that some versions of the GGI may be correlated with an increased ability of N. gonorrhoeae strains to cause systemic infection.

\section{THE GGI ENCODES A TYPE IV SECRETION SYSTEM}

The GGI contains 62 open reading frames, and many of these genes are homologous to T4SS genes (Hamilton et al., 2005; Figure 1). T4SSs have been identified in many Gram-negative and Grampositive bacteria, and include both conjugation systems as well as effector systems that translocate proteins or DNA-protein complexes (Christie, 2001; Alvarez-Martinez and Christie, 2009). The F-plasmid conjugation system of E. coli and the VirB/D T4SS of Agrobacterium tumefaciens are among the best characterized. A. tumefaciens uses the VirB/D system to secrete both proteins and oncogenic T-DNA directly into a plant cell, leading to the formation of crown gall tumors (Zhu et al., 2000). Many human pathogens also encode T4SSs that are involved in pathogenesis including Bordetella pertussis, which secretes pertussis toxin using a T4SS, and Legionella pneumophila, which secretes numerous effectors important for intracellular survival in macrophages (Shrivastava and Miller, 2009; Hubber and Roy, 2010). Expression of the cag T4SS in Helicobacter pylori results in the secretion of the virulence factor CagA as well as increased NF- $\kappa \mathrm{B}$ activation in host cells mediated by Nod 1 detection of peptidoglycan fragments (Viala et al., 2004; Backert and Selbach, 2008).

The GGI encodes 23 proteins with significant similarity to T4SS proteins, most of them contained in the first $28 \mathrm{~kb}$ of the island (Figure 1). Many of these genes show similarity to genes from the E. coli F-plasmid conjugation system, and the order of the genes in the GGI is highly similar to the IncF family of conjugative plasmids (Hamilton et al., 2005). There are several notable differences in gene organization between the GGI and the F-plasmid conjugation system, however, including $\operatorname{ltg} X$, which encodes a peptidoglycan transglycosylase similar to the geneX product (Orf169) from F-plasmid, as well as traD and traI, which encode the putative coupling protein and the relaxase, respectively (Frost et al., 1994; Hamilton et al., 2005). In the GGI, $\operatorname{ltg} X$ is the first gene in a predicted operon that contains the majority of the T4SS genes, while traI and traD are transcribed divergently (Hamilton et al., 2005; Figure 1). Interestingly, the relaxase encoded by the GGI is not phylogenetically related to the IncF family of relaxases but clusters instead with the IncH family of relaxases that includes relaxases from many integrative conjugative elements and genetic islands (Salgado-Pabón et al., 2007; Garcillan-Barcia et al., 2009).

In addition to the T4SS homologs, the GGI encodes 39 open reading frames, several of which are homologous to hypothetical genes (Hamilton et al., 2005). Some of these open reading frames do show sequence similarity to DNA-binding or processing proteins in sequence databases, including two DNA methylases (Ydg and YdhA), a helicase (Yea), a topoisomerase (TopB), and a singlestranded binding protein (Ssb; Hamilton et al., 2005). This region of the GGI has not been investigated as much as the region encoding the T4SS genes. However, this region might be a good place to look for as-yet unidentified protein substrates of the T4SS, additional unknown genes for T4S, or other factors that have caused the GGI to be maintained in $80 \%$ of gonococcal strains.

\section{THE GONOCOCCAL T4SS SECRETES CHROMOSOMAL DNA}

The gonococcal T4SS secretes chromosomal DNA into the extracellular environment in a contact-independent manner (Dillard and Seifert, 2001).Addition of DNaseI to the culture medium blocks the
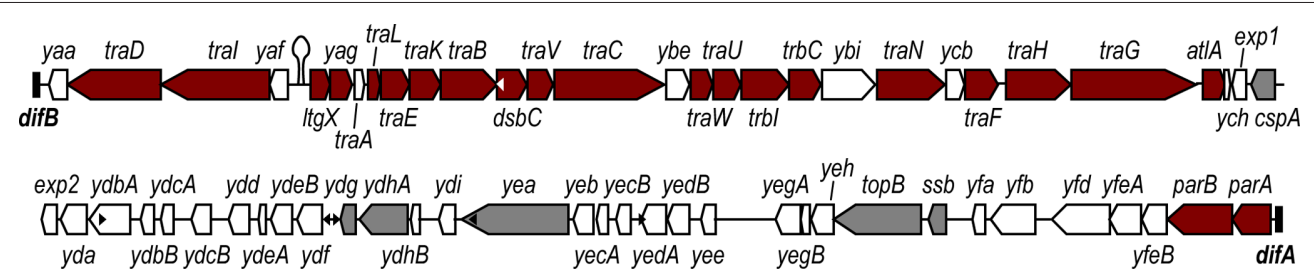

FIGURE 1 | Map of the gonococcal genetic island (GGI) from $\boldsymbol{N}$. gonorrhoeae strain MS11. The GGI in N. gonorrhoeae strain MS11 is $57 \mathrm{~kb}$ and is inserted in the chromosome at the dif site. The GGI encodes many homologs of type IV secretion system genes (red), as well as 33 genes of unknown function (white). There are six genes encoding homologs for DNA-binding or processing proteins (gray). In this map, traA is colored white despite encoding a homolog for the conjugative pilin subunit because it is not required for DNA secretion. The genes for the lytic transglycosylase AtIA and the putative peptidoglycan binding protein Yag are colored red because they are required for DNA secretion in gonococci even though they are not similar to known T4SS genes in other systems. The sequences of $\operatorname{tra} A$, traG, and at/A are variable between strains. The GGI contains six copies of the DNA uptake sequence (DUS, white and black triangles), significantly fewer copies than the flanking $57 \mathrm{~kb}$ of chromosomal DNA. A putative origin of transfer (oriT) has also been identified in the intergenic region between the yaf and ItgX genes (stem-loop). 
transfer of a genetic marker during co-culture, indicating that the secreted DNA is exposed to the extracellular environment and is not directly transferred between cells (Dillard and Seifert, 2001). Based on the susceptibility of the secreted DNA to different nucleases, it was determined that the DNA secreted by the gonococcal T4SS is single-stranded and blocked at the $5^{\prime}$ end (Salgado-Pabón et al., 2007). We hypothesize that the DNA remains bound to the relaxase at the $5^{\prime}$ end, as is the case for many other T4SSs (Young and Nester, 1988; Pansegrau et al., 1990). Although it is likely that the relaxase remains bound to the $5^{\prime}$ end of the secreted DNA, we currently have no direct evidence for protein secretion through the gonococcal T4SS. However, the secretion of proteins is highly probable. In fact, one model regarding DNA-secretory T4SSs is that they are actually protein secretion machines in which associated DNA comes along for the ride (Hazes and Frost, 2008). The contact-independent secretion of DNA by gonococci is unique among characterized T4SSs. Although B. pertussis secretes pertussis toxin directly into the extracellular environment, all other characterized T4SSs that transport DNA secrete it directly into a recipient or host cell (Cascales and Christie, 2003; Alvarez-Martinez and Christie, 2009).

Measurement of the transfer of a genetic marker from a donor to a recipient strain in co-culture has been used to identify some of the genes required for T4S in gonococci (Dillard and Seifert, 2001; Hamilton et al., 2001; Figure 2A). In this co-culture assay, wild-type donor strains are compared to donor strains with putative T4SS genes mutated. The donor strains also carry a chloramphenicol resistance $\left(\mathrm{Cm}^{\mathrm{R}}\right)$ marker and a mutation in either recA or pilT to ensure that they cannot also act as recipients in transformation. The donors are then co-cultured with a recipient strain that carries a spectinomycin resistance $\left(\mathrm{Sp}^{\mathrm{R}}\right)$ marker. After a period of 2-4 hours, bacteria from the co-culture are plated to enumerate $\mathrm{Cm}^{\mathrm{R}} \mathrm{Sp}^{\mathrm{R}}$ colonies. Co-culture with a donor carrying a mutation in a T4SS gene such as tra $G$ results in between 50 and 500-fold fewer $\mathrm{Cm}^{\mathrm{R}} \mathrm{Sp}^{\mathrm{R}}$ transformants than co-culture with a wild-type donor (Figure 2B; Dillard and Seifert, 2001; Hamilton et al., 2001).

Gonococci readily undergo autolysis, and it was previously assumed that DNA released by autolysis was the primary source of DNA for natural transformation (Hamilton and Dillard, 2006). However, in the co-culture assay described above, most of the transformation occurs during log-phase growth, suggesting that DNA donation by T4S is independent of autolysis (Dillard and Seifert, 2001). Indeed, various measures of autolysis indicate that T4SS mutants are not defective in autolysis. Cell viability is comparable between T4SS mutants and wild-type cells, and T4SS mutants release similar levels of both RNA and the cytoplasmic protein chloramphenicol acetyltransferase (CAT) compared to wild-type cells (Dillard and Seifert, 2001; Hamilton et al., 2005; Kohler et al., 2007). Furthermore, even if co-culture transformation is allowed to progress into stationary phase when autolysis occurs, DNA transfer occurs at a much higher frequency with a wild-type donor compared to a T4SS mutant donor (Dillard and Seifert, 2001). Thus, although DNA released by autolysis can contribute to transformation, DNA donation by the T4SS is independent of autolysis and appears to work better for transformation. It is unclear why this should be the case. Single-stranded DNA and double-stranded DNA transform gonococci with similar efficiency (Stein, 1991). Perhaps proteins bound to the secreted DNA encourage its uptake or its recombination during natural transformation.
Little is known about the regulation of DNA secretion in gonococci, and the GGI does not encode homologs of the regulatory factors that have been described for F-plasmid (Frost et al., 1994; Hamilton et al., 2005). Recently, however, it was shown that DNA secretion is increased in gonococci producing type IV pili, and that this increased secretion is due in part to increased expression levels of the relaxase TraI and the coupling protein TraD (Salgado-Pabón et al., 2010). Because traI and traD are divergently transcribed from the majority of the T4SS genes, differential regulation of these genes may allow regulation of DNA secretion (Salgado-Pabón et al., 2010). In gonococci, type IV pili are involved in DNA uptake as well as mediating host attachment and twitching motility (Swanson, 1973, 1978; Biswas et al., 1977). It is unclear why DNA secretion should be associated with piliation. One hypothesis is that DNA secretion may occur in response to signals released by piliated gonococci indicating the presence of competent members of the population (SalgadoPabón et al., 2010).

\section{THE PROTEINS OF THE GONOCOCCAL T4SS}

We propose a model of T4S in gonococci based on the similarity of the proteins encoded by the GGI with homologs from other characterized T4SSs (Figure 3). The T4SS proteins contained in the GGI fulfill several functions for T4S, including DNA processing, recruitment to the secretion apparatus, structural components of the apparatus, and possible pilus assembly.
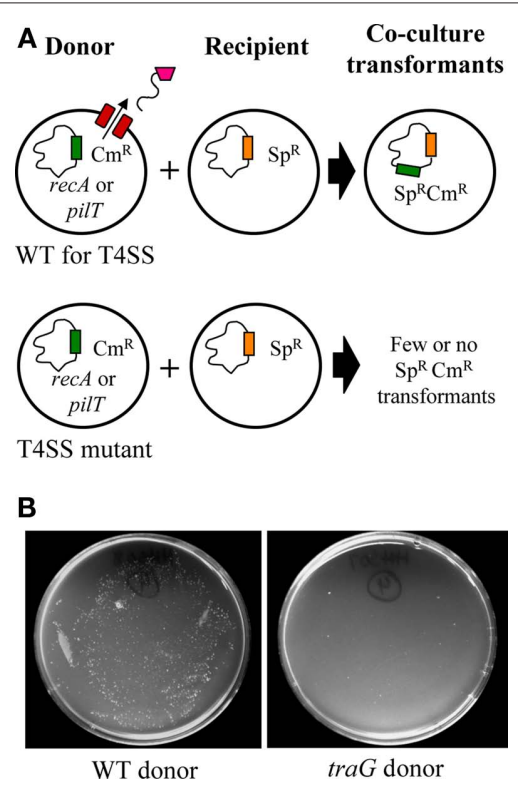

FIGURE 2 |The gonococcalT4SS secretes chromosomal DNA active in natural transformation. (A) To assay for DNA secretion, a donor strain that is either wild-type (WT) or mutant for various T4SS genes is grown in co-culture with a recipient strain. The donor strain carries a chloramphenicol resistance marker $\left(\mathrm{Cm}^{R}\right)$, while the recipient is marked with spectinomycin resistance $\left(\mathrm{Sp}^{\mathrm{R}}\right)$. The donor strain also carries a mutation in either pilT or recA to render it transformation-deficient and to ensure that it cannot act as a recipient. Following co-culture, bacteria are plated to double-selective plates to enumerate the number of $\mathrm{Cm}^{R} \mathrm{Sp}^{R}$ transformants. (B) Co-culture with a wild-type donor yields significantly more $\mathrm{Cm}^{R} \mathrm{Sp}^{R}$ colonies (left) compared to co-culture using a traG mutant donor (right). 


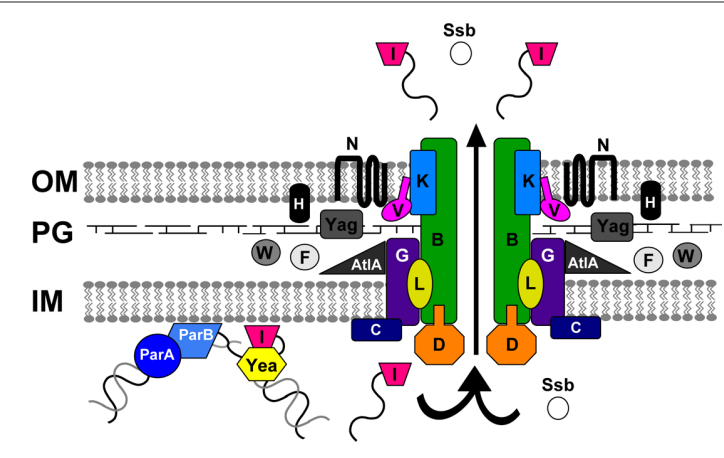

FIGURE 3 | Model of type IV secretion in gonococci. Predicted functions can be assigned to many of the proteins encoded by the GGI based on their similarity to other characterized type IV secretion system proteins. The putative partitioning proteins ParA and ParB may bring the DNA to the apparatus. Tral nicks the DNA at the origin of transfer (oriT), and a helicase, possibly Yea, unwinds it. The relaxase likely remains bound to the $5^{\prime}$ end of the DNA and pilots it to the secretion apparatus, where it may dock with the putative coupling protein, TraD. The putative ATPase TraC and the mating-pair stabilization homolog TraG likely contribute to the inner membrane pore. Periplasmic proteins such as Yag, TraW, TraF, TraH, and AtlA are likely involved in apparatus assembly or in making localized breaks in the peptidoglycan layer. The DNA is then secreted through the transmembrane apparatus, the core proteins of which are predicted to be TraB, TraK, and TraV. TraN may form part of the outer membrane pore. The DNA is secreted into the extracellular environment. Proteins such as single-stranded binding protein ( $\mathrm{Ssb}$ ) may also be secreted, but there is no evidence as-yet for protein secretion by the gonococcalT4SS. OM, outer membrane; PG, peptidoglycan; IM, inner membrane.

\section{DNA PROCESSING AND RECRUITMENT}

The proteins responsible for DNA processing and recruiting to the secretion apparatus have been well-studied in other T4SSs. In gonococci, DNA secretion likely begins when the DNA is brought into proximity of the T4SS machinery for processing. The GGI encodes homologs for both ParA and ParB, the partitioning proteins required for proper segregation of chromosomal and plasmid DNA during cell division (Leonard et al., 2005). In A. tumefaciens, a ParA-like protein (VirC1) is involved in localizing the T-DNA to the T4S apparatus for secretion (Leonard et al., 2005; Atmakuri et al., 2007). In the GGI, ParA, and ParB are separated from the majority of the T4SS genes by approximately $30 \mathrm{~kb}$ (Hamilton et al., 2005; Figure 1). However, ParA is essential for DNA secretion in gonococci, and a point mutation in the putative Walker box abolished DNA secretion (Hamilton et al., 2005). We hypothesize that ParA and ParB are required for bringing chromosomal DNA to the secretion apparatus where it can be processed by the relaxase.

Relaxases bind to and nick double-stranded DNA at a specific sequence known as the origin of transfer (oriT; Grinter, 1981). The reaction is mediated by one or two catalytic tyrosines and can result in the covalent attachment of the relaxase to the $5^{\prime}$ end of the DNA (Pansegrau et al., 1990). Previously characterized relaxases contain three motifs: the catalytic tyrosines, a motif facilitating DNAprotein interactions, and a histidine-rich motif involved in metal coordination (Pansegrau et al., 1994). Like most characterized relaxases, two tyrosines have been identified in gonococcal TraI that are required for efficient DNA secretion (Y93 and Y201). Mutation of Y93 completely blocks DNA transfer, while mutation of Y201 results in an intermediate level of transfer (Salgado-Pabón et al., 2007).
However, the GGI-encoded TraI differs significantly from other previously characterized relaxases. Point mutations altering the histidine-rich motif of $N$. gonorrhoeae TraI do not affect DNA secretion, suggesting that this motif may not be required for T4S in gonococci (Salgado-Pabón et al., 2007). N. gonorrhoeae TraI also differs from other relaxases by the presence of an HD phosphohydrolase domain. HD phosphohydrolase domains can sometimes coordinate metals, and some mutations in this region of gonococcal TraI result in diminished DNA secretion, suggesting that this domain may be fulfilling the role of the histidine-rich motif (Salgado-Pabón et al., 2007). Finally, N. gonorrhoeae TraI is distinguished from other characterized relaxases by the presence of an N-terminal hydrophobic region. This hydrophobic region is predicted to form an amphipathic helix, and it has been shown that this region enables TraI to associate with the cell membrane (Salgado-Pabón et al., 2007).

DNA processing by the relaxase occurs at a specific sequence known as the oriT. A single putative oriT was identified within the GGI between the genes yaf and $\operatorname{ltg} X$ (Figure 1) that exhibits many of the characteristics of previously characterized oriT sequences. The sequence contains an inverted repeat and is located near the relaxase gene in an $\mathrm{A}-\mathrm{T}$ rich region between divergent promoters (Lanka and Wilkins, 1995). An insertion disrupting this sequence reduced DNA secretion, while introduction of the inverted repeat region elsewhere on the chromosome restored DNA secretion. This result suggests that the oriT is located within the GGI between yaf and $\operatorname{ltg} X$ and that this region contains the only oriT on the gonococcal chromosome (Salgado-Pabón et al., 2007).

Once the DNA has been processed by the relaxase, the nucleoprotein complex must be targeted to the T4SS for secretion. This function is provided by coupling proteins - inner membrane ATPases that are widely conserved among T4SSs and couple the recognition of the substrate with the energy production necessary for translocation (de Paz et al., 2010). The GGI encodes the putative coupling protein $\mathrm{TraD}$, and deletion of $\mathrm{traD}$ diminished DNA secretion (Salgado-Pabón et al., 2010). Thus, in a model of gonococcal T4S, it is likely that ParA and ParB bring the DNA to the secretion apparatus where it is processed by TraI at the oriT contained on the chromosome within the GGI. We hypothesize that, as is the case for Hfr transfer in E. coli, the DNA would then be unwound by a helicase, possibly Yea, prior to secretion, and strand-replacement synthesis would regenerate the chromosome in the donor cell (Willetts and Wilkins, 1984). Since only one oriT is present, it is predicted that this region would be secreted first followed by the rest of the chromosome, with secretion of the chromosome proceeding in a unidirectional manner until the oriT is reached again.

\section{TRANSMEMBRANE APPARATUS}

Following processing and recruitment, the DNA substrate must pass through the T4SS apparatus, which forms a continuous channel across the inner and outer membranes (Fronzes et al., 2009a). The inner membrane pore likely consists of the ATPase TraC, the coupling protein $\mathrm{TraD}$, the mating-pair stabilization protein $\mathrm{TraG}$, and the N-terminal domain of TraB. Mutations in both traG and traD result in reduced DNA secretion, supporting the idea that these proteins may contribute to the T4SS apparatus in gonococci 
(Hamilton et al., 2001; Salgado-Pabón et al., 2010). The traG gene is highly variable between strains. In addition to the sac- 4 allele of $\operatorname{traG}$ that is correlated with isolates causing disseminated infection, an allele of $\operatorname{tra} G$ followed by a putative endopeptidase-encoding gene eppA has also been identified (Dillard and Seifert, 2001; Kohler et al., 2007). The function of TraG in T4S in gonococci remains unclear.

Our understanding of the structural biology of the core T4SS apparatus has greatly increased with the first cryo-electron tomography and crystal structures of a core T4SS apparatus from the IncN conjugative plasmid pKM101 (Chandran et al., 2009; Fronzes et al., 2009b). In these structures, the core T4S complex was composed of 14 copies each of the pKM101 homologs of TraK, TraV, and TraB (Chandran et al., 2009; Fronzes et al., 2009b). TraK homologs belong to a family of secretin-like proteins, while TraV homologs are lipoproteins required to stabilize the secretin. TraK and TraV homologs have been localized to the outer membrane in many other T4SSs and form a heterodimer linked by disulfide bonds (Baron et al., 1997; Harris et al., 2001). TraK and TraV also interact in gonococci, and we predict that these proteins also contribute to the outer membrane pore of the gonococcal T4SS (Hackett et al., 2008). In the crystal structure from pKM101, a two-helix bundle in the C-terminal domain of the TraB homolog was found to cross the outer membrane and was surface-exposed, making it the first characterized protein to insert in both the inner and outer membranes (Chandran et al., 2009). The two-helix bundle is conserved in gonococcal TraB, and we predict that this portion of TraB may also be surface-exposed (our unpublished observation).

Because N. gonorrhoeae and N. meningitidis can only be found within human hosts, it is noteworthy that any surface-exposed proteins in the T4SS could be exposed to the immune system of the host. Predicted surface-exposed proteins include TraK and TraB, as well as the protein TraN, which plays a role in stabilizing interactions between mating-pairs in E. coli F-plasmid (Lawley et al., 2003). Since gonococci secrete DNA in a contact-independent manner, the formation of mating-pairs is unnecessary, and it is unclear what role TraN might be playing in gonococci. However, mutation of $\operatorname{traN}$ greatly reduces DNA secretion in gonococci, indicating that TraN is required for T4S (Hamilton et al., 2005). Many surfaceexposed proteins in Neisseria undergo high-frequency antigenic or phase variation, including the opacity proteins, pilin, and porin (Stern et al., 1986; Gibbs et al., 1989; Fudyk et al., 1999; Hill and Davies, 2009). However, based on eight sequenced gonococcal genomes that contain the GGI as well as sequence data from two additional gonococcal strains, predicted surface-exposed T4SS proteins such as TraN, TraK, and TraB show only minor sequence differences between strains, suggesting that these proteins may be rare conserved surface proteins in Neisseria (GenBank accession no. CP001050 and http://www.broadinstitute.org/annotation/genome/ neisseria_gonorrhoeae/GenomesIndex.html; Hackett et al., 2008).

\section{PILUS ASSEMBLY}

Type IV secretion systems such as E. coli F-plasmid or the A. tumefaciens VirB/D system construct conjugative pili (Kado, 2000; Lawley et al., 2003). The importance of the conjugative pilus in DNA transfer is unclear, although several models have been proposed. An early model proposed that the pilus could serve as a conduit for the DNA to travel between donor and recipient. Alternatively, the retraction of the pilus could facilitate close contact between the donor and recipient and allow the direct transfer of DNA (Kado, 2000). An interesting new hypothesis suggests that the pilus may help the donor cell to "sample" the surrounding environment for potential recipients (Silverman and Clarke, 2010). The GGI encodes a homolog for the pilin subunit $\operatorname{TraA}$ (Figure 1). The traA sequence is variable, with both a short allele and a long allele present in Neisseria strains, and the GGI in the gonococcal strain MS11 contains the short allele (Jain et al., 2008; Llosa et al., 2009). We predict that the longer allele encodes the full-length pilin protein, while the short allele may encode a pilin that cannot be assembled. However, TraA is not required for T4S in gonococci, since deletion of traA has no effect on DNA secretion (Immel et al., 2003). If the role of the conjugative pilus is to facilitate close contact between a donor and a recipient cell, then a pilus may not be necessary for gonococcal T4S since the DNA is secreted into the extracellular environment and the donor does not require contact with the recipient for natural transformation.

\section{THE GGI IN N. MENINGITIDIS}

When the GGI was first identified in N. gonorrhoeae, attempts were also made to detect the GGI in a panel of symbiotic Neisseria species and N. meningitidis using PCR or low-stringency Southern blotting for $\operatorname{tra} G$ and atlA. However, $\operatorname{tra} G$ and atlA are variable in the GGI, and it was not detected (Dillard and Seifert, 2001). The GGI was also absent in the genome sequences for meningococcal serotype A and B strains that had been published. Thus, it was unexpected when versions of the GGI were identified in meningococci in 2005 (Snyder et al., 2005). Snyder et al. (2005) used meningococcal chromosomal DNA to probe a microarray containing a single oligonucleotide for each gene in the GGI. Six meningococcal strains were found to have the GGI, and three island types were represented among the strains. No meningococcal strain had a GGI identical to that of gonococcal strain MS11, and three of the strains had large deletions of T4SS genes. The variant GGIs were found in serotype $\mathrm{H}, \mathrm{W}-135$, and Z strains. Although it appears the GGI is not present in most strains of N. meningitidis, it will be interesting to learn what portion of the meningococcal population carries a version of the GGI and whether it is present in strains from sequence types common in invasive disease or symbiotic colonization.

Recently, a draft genome from meningococcal strain $\alpha 275$, which contains the GGI, has become available (GenBank accession no. AM889138). Analysis of the sequence shows that the GGI from this strain is $64 \mathrm{~kb}$ and differs from the GGI in N. gonorrhoeae strain MS11 in several ways (Figure 4). The GGI in $\alpha 275$ contains the long allele of $\operatorname{traA}$, a variant allele of $\operatorname{tra}$, and the putative endopeptidase-encoding gene, eppA. Each of these variations is also present in some gonococcal strains. Additionally, a deletion from within $t r a D$ results in the truncation of $t r a D$, and $\operatorname{dif} B$ being contained within the $t r a D$ coding region. Another significant difference is the presence of an insertion of IS1655 just after the start codon of traK. Analysis of the inserted sequence did not reveal a possible ribosome binding site or in-frame start for the interrupted traK (our unpublished observation). As a result of the insertion in $t r a K$ and deletion in $t r a D$, it is unlikely that $\alpha 275$ is capable of making a functional T4SS or secreting DNA. 


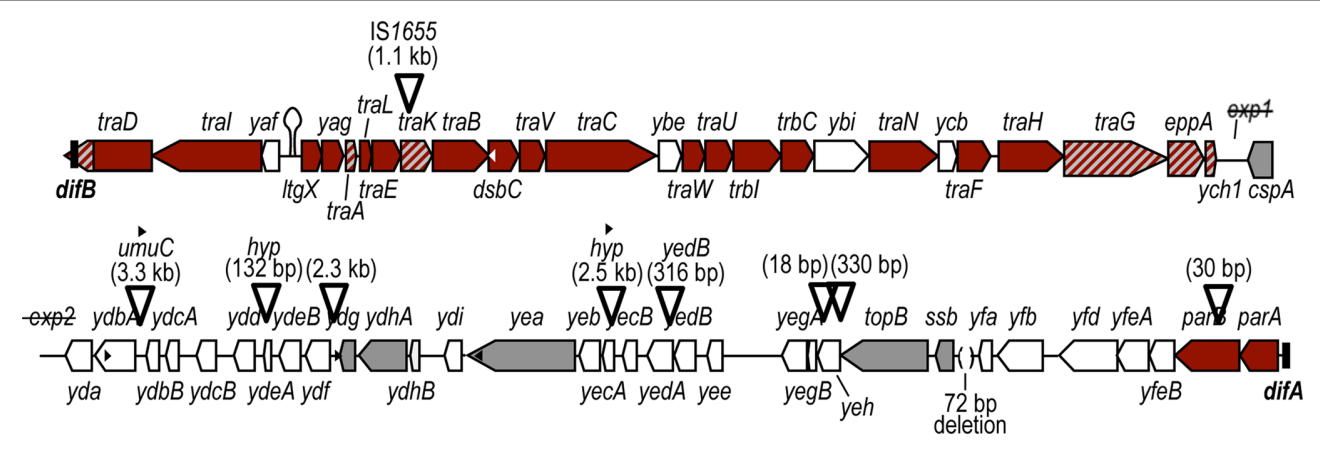

FIGURE 4 | Map of the GGI from $\boldsymbol{N}$. meningitidis strain $\boldsymbol{\alpha 2 7 5}$. The GGI in $\alpha 275$ is $64 \mathrm{~kb}$ and differs in several ways from the GGI in N. gonorrhoeae MS11. Insertions in the $\alpha 275 \mathrm{GGl}$ are indicated by inverted triangles, with the text above the triangle indicating both the size of the insertion as well as the identity of the sequence, if known. Two insertions showed similarity to hypothetical genes from N. gonorrhoeae, N. meningitidis, N. mucosa, and N. flava (hyp). If no sequence is indicated above a triangle, the insertion is not similar to any sequence in GenBank. Genes that are significantly different from homologs in the GGI of N. gonorrhoeae MS11 are indicated by red and gray diagonal lines. In $\alpha 275$, a deletion in tra $D$ results in the truncation of tra $D$, and difB is contained within the traD coding region. This GGI contains the long allele of traA, and IS 1655 is inserted just after the start codon of traK. A truncated allele of traG is present followed by the hypothetical endopeptidase eppA (also found in some gonococci). There is a deletion of exp1. The $\alpha 275$ $\mathrm{GGI}$ also has an insertion of partial DNA polymerase $V$ subunit umuC at $y d b B$ and an insertion of partial yedB at yedA. A small deletion upstream of $s s b$ is indicated by parentheses. DNA uptake sequences (DUS) are indicated by small black or white triangles. In addition to the four DUS indicated in the figure, two DUS are located in the inserted sequences (black triangles over the inserted sequence).
Based on the sequence of the GGI in $\alpha 275$ and the hybridization studies of Snyder et al. (2005), it appears that several of the meningococcal GGIs are degrading, acquiring deletions and insertions. It is unlikely that they encode functional secretion systems. However, three of the GGIs identified in meningococci do carry most of the T4SS genes and might yet produce a functional T4SS (Snyder et al., 2005). It is unclear whether these secretion systems would function for DNA secretion as the T4SS does in gonococci. None of the meningococcal GGIs carry atlA, which encodes a peptidoglycanase essential for DNA secretion in gonococci (Dillard and Seifert, 2001; Kohler et al., 2007). One of the three mostly intact GGI variants has parB deleted, a gene which would be expected to be required for DNA secretion, since a functional ParA is required (Hamilton et al., 2005). Contrary to what was reported by Snyder et al. (2005) in their hybridization studies, PCR analysis and sequencing data indicate that all of the meningococcal GGIs do encode ParA (our unpublished observation). It is possible that these T4SSs may have some function other than DNA secretion. They could secrete proteins, or the apparatus might serve an adhesive function, as was seen for a T4SS in Bartonella (Vayssier-Taussat et al., 2010).

\section{THE T4SS ALLOWS TON-INDEPENDENT SURVIVAL OF GONOCOCCI DURING INTRACELLULAR INFECTION}

The Ton complex, consisting of TonB, ExbB, and ExbD, is required for iron transport into both gonococci and meningococci. Previous work in gonococci and meningococci established that the Ton complex is required for intracellular survival during epithelial cell infection (Larson et al., 2002; Hagen and Cornelissen, 2006). However, Zola et al. (2010) found that gonococcal strain MS11 was able to survive intracellularly without a functional Ton complex. A strain of MS11 deleted for the GGI and without a functional Ton complex was not able to survive intracellular infection. Intracellular survival could be restored by addition of iron to the medium, confirming that it was the inability of the bacteria to acquire iron that resulted in the bacterial death. Experiments using strains with mutations in individual T4SS genes demonstrated that the T4SS apparatus is required for Ton-independent survival, but DNA secretion is not required (Zola et al., 2010). Although the mechanism of survival is unknown, several hypotheses could explain these results. First, it is possible that the T4SS secretes an unknown factor that is able to bind iron so that it can be taken up by gonococci or stimulate the release of iron sequestered by the host (Figure 5A). Second, iron could be entering the cell through the T4SS apparatus (Figure 5B). There are precedents for molecules sneaking in through such apparatuses. Antibiotics and heme can enter gonococci through the pilus pore in certain pilQ variants (Chen et al., 2004). Also, the T4SS of L. pneumophila makes the bacteria sensitive to salt in the medium (Sadosky et al., 1993). The third hypothesis is that the T4SS could affect gonococcal trafficking within epithelial cells so that cells expressing the T4SS traffic to an iron-rich niche (Figure 5C). Other bacteria use T4SSs to interact with and affect host cell trafficking, so it is possible that the gonococcal T4SS is serving a similar function (Isberg et al., 2009).

\section{THE GONOCOCCAL GENETIC ISLAND AS A MOBILE ELEMENT}

The GGI shows evidence of horizontal acquisition from another bacterial species, and it appears that the bacterium's own sitespecific recombinase was responsible for incorporating this foreign DNA into the genome (Domínguez et al., 2011). The data from several experiments suggest that the site-specific recombinase XerCD integrated the GGI into the gonococcal chromosome. The direct repeats flanking the GGI, difA and $\operatorname{dif} B$, are both recognition sites for XerCD, with difA being a consensus sequence and difB having four mismatches to the consensus (Hamilton et al., 2005; Domínguez et al., 2011). The function of XerCD is usually to resolve chromosomal dimers that arise during replication. During chromosome replication homologous recombination can occur between the daughter chromosomes resulting in the two chromosomes being linked as a single molecule, a head-to-tail dimer. XerCD mediates a site-specific recombination reaction that separates the 
A

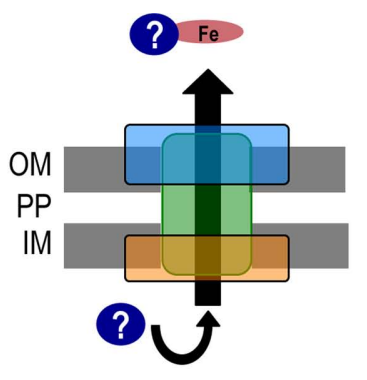

B

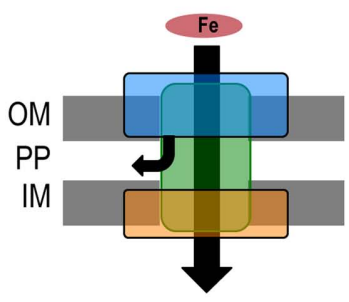

C

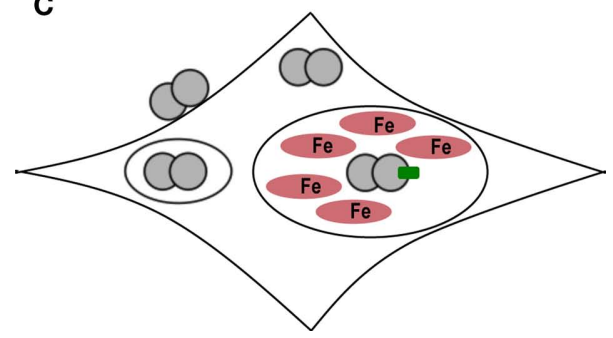

FIGURE 5 | Potential methods of Ton-independent iron acquisition. (A) The type IV secretion system could secrete a factor that either binds iron or stimulates iron release by the host, thereby facilitating iron uptake by gonococci. (B) The T4SS apparatus may act as a pore for iron to pass into either the periplasm or the cytosol. The T4SS pore is represented in a simplified fashion by the outer membrane protein complex (blue rectangle), the transmembrane proteins (green rectangle), and the inner membrane protein complex (orange rectangle). (C) The T4SS could affect gonococcal trafficking within host epithelial cells so that gonococci expressing aT4SS (diplococcus with green box) go to an iron-rich niche. OM, outer membrane; PP, periplasm; IM, inner membrane.

chromosomes (Carnoy and Roten, 2009). A similar type of reaction can occur in a chromosome that carries the GGI. XerCD can act at the two dif sites to excise the GGI from the chromosome, creating a circular form of the GGI. However, the reaction can also go in the opposite direction, and XerCD can integrate DNA into the chromosome if the DNA carries a dif site. This type of integration was demonstrated for gonococci by cloning the difA site plus $90 \mathrm{bp}$ from the GGI and transforming the resulting plasmid into a gonococcal strain that did not carry the GGI. The plasmid integrated into the chromosome at the dif site (Hamilton et al., 2005). We hypothesize that the GGI was similarly acquired, that it was introduced into gonococci as a plasmid, and like some known plasmids (Blakely et al., 1991), it carried a dif-like site. XerCD could then have integrated it into the chromosome where it became mostly stabilized through acquisition of mutations at $\operatorname{dif} B$.

Mutations at $\operatorname{dif} B$ and mutation of $x e r D$ demonstrated the involvement of the dif-XerCD system in GGI recombination. In strain MS11, the GGI was found to be lost from the cells at a frequency of $10^{-6}$ after $18 \mathrm{~h}$ of growth. However, if the dif $B$ sequence was mutated to a consensus difA sequence (resulting in the GGI being flanked by two consensus difA sequences), then the GGI was lost at a frequency of $10^{-3}$. By contrast, a mutation in $x e r D$ prevented GGI loss even in the strain with two difA flanking sequences (Domínguez et al., 2011). These results suggest that XerD is necessary for GGI excision and loss and that increased similarity of the GGI-flanking sequences to the XerCD binding sequences facilitates higher frequency excision and loss of the GGI. The loss of the GGI from cells in the population indicates that the GGI cannot replicate on its own, or if it can, it does so poorly. PCR analyses to detect the GGI as a circle could easily detect the circle in the double $\operatorname{dif} A^{+}$strain but could barely detect the circle in the wild-type strain, and then only after a high number of amplification cycles (Domínguez et al., 2011). However, the GGI was easily detected in the chromosome. Thus, the GGI is nearly always in the chromosome and only rarely excises to form an extrachromosomal circle.

It is possible that the ability of the GGI to circularize could allow it to be transferred to another bacterium. When the GGI is secreted by the T4SS as part of the chromosome, the oriT within the GGI is the first region to be secreted, and it is not until the entire chromosome has been secreted that the remainder of the GGI is exported. However, if the GGI excises from the chromosome, then the GGI could be secreted as a smaller DNA molecule. It would still be difficult for the GGI to be transferred as this smaller molecule, since it would need to be taken up in its entirety and re-circularized in the recipient. The recircularization might not be as unlikely as it would first seem, however, since relaxases of conjugative plasmids perform such a reaction in the recipient, and the gonococcal TraI might do so as well (Garcillan-Barcia et al., 2007).

It is curious that all gonococci examined have been found to have the same $\operatorname{dif} B$ sequence with the same four mismatches compared to the consensus dif (Domínguez et al., 2011). If the utility of sequence changes in dif is to stabilize the GGI in the chromosome, why are the same four changes always present? Different mismatches are present in $N$. meningitidis, so it is unlikely that these mismatches were present in an ancestral Neisseria species that acquired the GGI. Perhaps excision of the GGI could sometimes be advantageous (at least for GGI propagation if not for N. gonorrhoeae itself), and the difB sequence is an escape hatch. The GGI would be maintained and replicated in the chromosome most of the time but occasionally could be excised and transferred.

Unlike the GGI in N. gonorrhoeae, some versions of the GGI appear to be stuck in N. meningitidis chromosomes and are unable to excise. The difB2 allele is found in some N. meningitidis strains and has eight mismatches compared to the dif consensus (Domínguez et al., 2011). The difB2 sequence appears to have arisen from a deletion occurring between dif $B$ and the traD coding sequence. It is unlikely that this dif sequence would function for XerCD-mediated recombination. The inability of the GGI to excise in these strains may explain why some $N$. meningitidis strains maintain a GGI that is unlikely to produce a functional secretion system.

\section{CONCLUSION}

The GGI is a large, horizontally acquired genomic island present in most strains of N. gonorrhoeae and in some strains of N. meningitidis. As is the case for many horizontally transferred genetic 
islands in bacteria, the origin of the GGI is unknown. However, since $N$. gonorrhoeae and N. meningitidis are found exclusively inside a human host, we hypothesize that the GGI was acquired from another human colonizer, just as Haemophilus genes have been found to be acquired by Neisseria (Kroll et al., 1998). The GGI is not similar to conjugative plasmids found in some gonococcal strains (Pachulec and van der Does, 2010). In gonococci, the GGIencoded T4SS acts to secrete chromosomal DNA. This DNA secretion likely increases DNA transfer in the gonococcal population, increasing genetic diversity, and creating new recombinants that may avoid the host immune response. DNA transfer through the T4SS may also facilitate the spread of antibiotic resistance genes, a continual problem for the treatment of gonorrhea. The mechanism of transfer appears similar to that of a conjugation system or $\mathrm{Hfr}$ transfer in E. coli. The chromosome is cut at a single site (oriT) by a relaxase, TraI, unwound, and transported as a single-strand through the T4SS, with DNA at the oriT transferred first, followed by the rest of the chromosome in an ordered fashion. An important difference between the gonococcal T4SS and conjugation systems is that in gonococci, DNA is secreted into the surrounding milieu and is not transferred directly from cell to cell. It is likely that the T4SS also transports other substrates in addition to DNA, such as proteins, but none have yet been identified.

There are many unanswered questions regarding the role of the gonococcal T4SS in host-pathogen interactions. It is clear from the TonB experiments that the T4SS is expressed when gonococci are intracellular. However, the T4SS may be expressed at other times during infection as well, and it is unclear what impact the T4SS might have on gonococcal pathogenesis or biology under these conditions. For example, the fact that certain forms of the GGI are correlated with disseminated infections may suggest that there is a role for T4S during systemic infection. Although secreted protein

\section{REFERENCES}

Alvarez-Martinez, C. E., and Christie, P. J. (2009). Biological diversity of prokaryotic type IV secretion systems. Microbiol. Mol. Biol. Rev. 73, 775-808.

Atmakuri, K., Cascales, E., Burton, O. T., Banta, L. M., and Christie, P. J. (2007). Agrobacterium ParA/MinD-like VirC1 spatially coordinates early conjugative transfer reactions. EMBO J. 26, 2540-2551.

Backert, S., and Selbach, M. (2008). Role of type IV secretion in Helicobacter pylori pathogenesis. Cell. Microbiol. 10, 1573-1581.

Baron, C., Thorstenson, Y. C., and Zambryski, P. C. (1997). The lipoprotein VirB7 interacts with VirB9 in the membranes of Agrobacterium tumefaciens. J. Bacteriol. 179, 1211-1218.

Biswas, G. D., Sox, T., Blackman, E., and Sparling, P. F. (1977). Factors affecting genetic transformation of Neisseria gonorrhoeae. J. Bacteriol. 129, 983-992.

Blakely, G., Colloms, S., May, G., Burke, M., and Sherratt, D. (1991). Escherichia coli XerC recombinase is required for chromosomal segregation at cell division. New Biol. 3, 789-798.
Carnoy, C., and Roten, C. (2009). The dif/Xer recombination systems in proteobacteria. PLoS ONE 4, e6531. doi: 10.1371/journal.pone.0006531

Cascales, E., and Christie, P. J. (2003). The versatile bacterial type IV secretion systems. Nat. Rev. Microbiol. 1, 137-149.

Chandran, V., Fronzes, R., Duquerroy, S., Cronin, N., Navaza, J., and Waksman, G. (2009). Structure of the outer membrane complex of a type IV secretion system. Nature 462, 1011-1015.

Chen, C. J., Tobiason, D. M., Thomas, C. E., Shafer, W. M., Seifert, H. S., and Sparling, P. F. (2004). A mutant form of the Neisseria gonorrhoeae pilus secretin protein PilQ allows increased entry of heme and antimicrobial compounds. J. Bacteriol. 186, 730-739.

Christie,P.J.(2001).Type IV secretion: intercellular transfer of macromolecules by systems ancestrally related to conjugation machines. Mol. Microbiol. 40, 294-305.

de Paz, H. D., Larrea, D., Zunzunegui, S., Dehio, C., Cruz, F. d. 1., and Llosa, M. (2010). Functional dissection of the conjugative coupling protein TrwB. J. Bacteriol. 192, 2655-2669.

Dillard, J. P., and Seifert, H. S. (2001). A variable genetic island specific for

effectors have not yet been identified, it is easy to imagine that such effectors might be involved in host immune modulation, intracellular trafficking, nutrient acquisition, or other functions that enable gonococci to be so well-adapted for life in a human host. Regardless of whether the T4SS secretes proteins in addition to a DNA-protein complex, however, any transported substrates will necessarily come in contact with host cells and may affect gonococcal infection. The secreted DNA could possibly be recognized by pattern recognition receptors, thereby inducing an inflammatory response. Additionally, the secreted DNA may affect binding or other interactions of gonococci with each other or with host cells.

Dissecting the role of T4S in gonococcal pathogenesis is an important topic for ongoing research, and as we continue to develop tools for studying the T4SS in the pathogenic Neisseria, such as antibodies to T4SS proteins and strains with regulated expression of the T4SS genes, we will be better able to address this question. The role of the GGI in N. meningitidis also remains a mystery and an important topic of investigation. In some meningococcal strains, the GGI is a scrap-heap of mutated genes. In other strains, many of the T4SS genes are intact, but genes needed for DNA secretion are missing. In these cases, it is still possible that the T4SS in meningococci may secrete proteins or that the T4SS may have some other role in infection. Understanding how the GGI is affecting both Neisseria populations and the function of the individual cell will lead us to a better understanding of $N$. gonorrhoeae and $N$. meningitidis horizontal gene transfer and pathogenesis.

\section{ACKNOWLEDGMENTS}

We thank Holly L. Hamilton for performing the co-culture assay shown in Figure 2. Work in the Dillard laboratory is supported by grants NIH AI047958 and AI072605 to Joseph P. Dillard. Meghan E. Ramsey is supported by traineeship on NIH T32 GM07215.

Neisseria gonorrhoeae is involved in providing DNA for natural transformation and is found more often in disseminated infection isolates. $\mathrm{Mol}$. Microbiol. 41, 263-278.

Domínguez, N. M., Hackett, K. T., and Dillard, J. P. (2011). XerCD-mediated site-specific recombination leads to loss of the 57-kilobase gonococcal genetic island. J. Bacteriol. 193, 377-388.

Fronzes, R., Christie, P. J., and Waksman, G. (2009a). The structural biology of type IV secretion systems. Nat. Rev. Microbiol. 7, 703-714.

Fronzes, R., Schafer, E., Wang, L., Saibil, H. R., Orlova, E. V., and Waksman, G. (2009b). Structure of a type IV secretion system core complex. Science 323, 266-268.

Frost, L. S., Ippen-Ihler, K., and Skurray, R. A. (1994). Analysis of the sequence and gene products of the transfer region of the F sex factor. Microbiol. Rev. 58, $162-210$.

Fudyk, T., Maclean, I., Simonsen, J., Njagi, E., Kimani, J., Brunham, R., and Plummer, F. (1999). Genetic diversity and mosaicism at the por locus of Neisseria gonorrhoeae. J. Bacteriol. 181, 5591-5599.
Gal-Mor, O., and Finlay, B. B. (2006). Pathogenicity islands: a molecular toolbox for bacterial virulence. Cell. Microbiol. 8, 1707-1719.

Garcillan-Barcia, M. P., Franci, M. V., and de la Cruz, F. (2009). The diversity of conjugative relaxases and its application in plasmid classification. FEMS Microbiol. Rev. 33, 657-687.

Garcillan-Barcia, M. P., Jurado, P., Gonzalez-Perez, B., Moncalian, G. Fernandez, L. A., and Cruz, F. d. 1. (2007). Conjugative transfer can be inhibited by blocking relaxase activity within recipient cells with intrabodies. Mol. Microbiol. 63, 404-416.

Gibbs, C., Reimann, B., Schultz, E., Kaufmann, A., Haas, R., and Meyer, T. (1989). Reassortment of pilin genes in Neisseria gonorrhoeae occurs by two distinct mechanisms. Nature 338, 651-652.

Goodman, S. D., and Scocca, J. J. (1988). Identification and arrangement of the DNA sequence recognized in specific transformation of Neisseria gonorrhoeae. Proc. Natl. Acad. Sci. U.S.A. 85, 6982-6986.

Grinter, N. J. (1981). Analysis of chromosome mobilization using hybrids 
between plasmid RP4 and a fragment of bacteriophage lambda carrying IS1. Plasmid 5, 267-276.

Hacker, J., and Kaper, J. B. (2000). Pathogenicity islands and the evolution of microbes. Annu. Rev. Microbiol. 54, 641-679.

Hackett, K. T., Hamilton, H. L., and Dillard, J. P. (2008). "Determining the surface components of the type IV secretion system in Neisseria gonorrhoeae and Neisseria meningitidis," in Abstracts of the 108th General Meeting of the American Society for Microbiology, Boston, MA.

Hagen, T. A., and Cornelissen, C. N. (2006). Neisseria gonorrhoeae requires expression of TonB and the putative transporter TdfF to replicate within cervical epithelial cells. Mol. Microbiol. 62, 1144-1157.

Hamilton, H. L., and Dillard, J. P. (2006). Natural transformation of Neisseria gonorrhoeae: from DNA donation to homologous recombination. Mol. Microbiol. 59, 376-385.

Hamilton, H. L., Domínguez, N. M., Schwartz, K. J., Hackett, K. T., and Dillard, J. P. (2005). Neisseria gonorrhoeae secretes chromosomal DNA via a novel type IV secretion system. Mol. Microbiol. 55, 1704-1721.

Hamilton, H. L., Schwartz, K. J., and Dillard, J. P. (2001). Insertionduplication mutagenesis of Neisseria: use in characterization of DNA transfer genes in the gonococcal genetic island. J. Bacteriol. 183, 4718-4726.

Harris, R. L., Hombs, V., and Silverman, P. M. (2001). Evidence that F-plasmid proteins TraV, TraK and TraB assemble into an envelope-spanning structure in Escherichia coli. Mol. Microbiol. 42, 757-766.

Hazes, B., and Frost, L. (2008). Towards a systems biology approach to study type II/IV secretion systems. Biochim. Biophys. Acta 1778, 1839-1850.

Hill, S. A., and Davies, J. K. (2009). Pilin gene variation in Neisseria gonorrhoeae: reassessing the old paradigms. FEMS Microbiol. Rev. 33, 521-530.

Hubber, C., and Roy, C. R. (2010). Modulation of host cell function by Legionella pneumophila type IV effectors. Annu. Rev. Cell Dev. Biol. 26, 261-283.

Immel, S. K., Hamilton, H. L., and Dillard, J. P. (2003). "Mutagenesis of novel genes in the type IV secretion system region of the gonococcal genetic island," in Abstracts of the 103rd General Meeting of the American Society for Microbiology, Washington, DC, 227 .

Isberg, R. R., O'Connor, T. J., and Heidtman, M. (2009). The Legionella pneumophila replication vacuole: making a cosy niche inside host cells. Nat. Rev. Microbiol. 7, 13-24.
Jain, S., Pachulec, E., and Does, C. v. d. (2008). "Assembly of the pilus of the type IV DNA secretion machinery of Neisseria gonorrhoeae," in Abstracts of the Current Trends in Biomedicine Workshop: Bacterial Type IV Secretion Systems in Human Disease, Baeza.

Kado, C. I. (2000). The role of the T-pilus in horizontal gene transfer and tumorigenesis. Curr. Opin. Microbiol. 3, 643-648.

Karlin, S. (1998). Global dinucleotide signatures and analysis of genomic heterogeneity. Curr. Opin. Microbiol. 1, 598-610.

Kohler, P. L., Hamilton, H. L., CloudHansen, K., and Dillard, J. P. (2007). AtlA Functions as a peptidoglycan lytic transglycosylase in the Neisseria gonorrhoeae type IV secretion system. J. Bacteriol. 189, 5421-5428.

Kroll, J. S., Wilks, K. E., Farrant, J. L., and Langford, P. R. (1998). Natural genetic exchange between Haemophilus and Neisseria: intergeneric transfer of chromosomal genes between major human pathogens. Proc. Natl. Acad. Sci. U.S.A. 95, 12381-12385.

Lanka, E., and Wilkins, B. M. (1995). DNA processing reactions in bacterial conjugation. Annu. Rev. Biochem. 64, 141-169.

Larson, J. A., Higashi, D. L., Stojiljkovic, I., and So, M. (2002). Replication of Neisseria meningitidis within epithelial cells requires TonB-dependent acquisition of host cell iron. Infect. Immun. 70, 1461-1467.

Lawley, T. D., Klimke, W. A., Gubbins, M. J., and Frost, L. S. (2003). F factor conjugation is a true type IV secretion system. FEMS Microbiol. Lett. 224, 1-15.

Leonard, T. A., Moller-Jensen, J., and Lowe, J. (2005). Towards understanding the molecular basis of bacterial DNA segregation. Philos. Trans. R. Soc. Lond. B Biol. Sci. 360, 523-535.

Llosa, M., Roy, C., and Dehio, C. (2009). Bacterial type IV secretion systems in human disease. Mol. Microbiol. 73, 141-151.

McShan, W. M., Williams, R. P., and Hull, R. A. (1987). Recombinant molecule from a disseminating strain of Neisseria gonorrhoeae that confers serum bactericidal resistance. Infect. Immun. 55, 3017-3022.

Nowicki, S., Ram, P., Pham, T., Goluszko, P., Morse, S., Anderson, G. D., and Nowicki, B. (1997). Pelvic inflammatory disease isolates of Neisseria gonorrhoeae are distinguished by Clqdependent virulence for newborn rats and by the sac- 4 region. Infect. Immun . 65, 2094-2099.

Pachulec, E., and van der Does, C. (2010). Conjugative plasmids of Neisseria gonorrhoeae. PLoS ONE 5, e9962. doi: 10.1371/journal.pone.0009962

Pansegrau, W., Shroder, W., and Lanka, E. (1994). Concerted action of three distinct domains in the DNA cleavingjoining reaction catalyzed by relaxase (TraI) of conjugative plasmid RP4. J. Biol. Chem. 269, 2782-2789.

Pansegrau, W., Zieglin, G., and Lanka, E. (1990). Covalent association of the tral gene product of plasmid RP4 with the 5 -terminal nucleotide at the relaxation nick site. Proc. Natl. Acad. Sci. U.S.A. 87, 6555-6559.

Sadosky, A. B., Wiater, L. A., and Shuman, H. A. (1993). Identification of Legionella pneumophila genes required for growth within and killing of human macrophages. Infect. Immun. 61, 5361-5373.

Salgado-Pabón, W., Du, Y., Hackett, K. T., Lyons, K. M., Arvidson, C. G., and Dillard, J. P. (2010). Increased expression of the type IV secretion system in piliated Neisseria gonorrhoeae. J. Bacteriol. 192, 1912-1920.

Salgado-Pabón, W., Jain, S., Turner, N., van der Does, C., and Dillard, J.P. (2007). A novel relaxase homologue is involved in chromosomal DNA processing for type IV secretion in Neisseria gonorrhoeae. Mol. Microbiol. 66, 930-947.

Shrivastava, R., and Miller, J. F. (2009). Virulence factor secretion by Bordetella species. Curr. Opin. Microbiol. 12 88-93.

Silverman, P.M., and Clarke, M. B. (2010), New insights into F-pilus structure, dynamics, and function. Integr. Biol. 2, 25-31.

Smith, H. O., Gwinn, M. L., and Salzberg, S. L. (1999). DNA uptake signal sequences in naturally transformable bacteria. Res. Microbiol. 150 603-616.

Snyder, L.A., Jarvis, S. A., and Saunders, N. J. (2005). Complete and variant forms of the "gonococcal genetic island" in Neisseria meningitidis. Microbiology 151, 4005-4013.

Stein, D. C. (1991). Transformation of Neisseria gonorrhoeae: physical requirements of the transforming DNA. Can. J. Microbiol. 37, 345-349.

Stern, A., Brown, M., Nickel, P., and Meyer, T. F. (1986). Opacity genes in Neisseriagonorrhoeae: control of phase and antigenic variation. Cell 47,61-71.

Swanson, J. (1973). Studies on gonococcus infection. IV. Pili: their role in attachment of gonococci to tissue culture cells. J. Exp. Med. 137, 571-589.

Swanson, J. (1978). Studies on gonococcus infection. XII. Colony color and opacity variants of gonococci. Infect. Immun. 19, 320-331.

Vayssier-Taussat, M., Rhun, D. L., Deng, D. K., Biville, F., Cescau, S., Danchin, A., Marignac, G., Lenaour, E., Boulouis, H. J., Mavris, M., Arnaud, L., Yang, H., Wang, J., Quebatte, M., Engel, P., Saenz, H., and Dehio, C. (2010). The Trw type IV secretion system of Bartonella mediates host-specific adhesion to erythrocytes. PLoS Pathog. 6:e1000946. doi: 10.1371/journal. ppat. 1000946

Viala, J., Chaput, C., Boneca, I. G., Cardona, A., Giardin, S. E., Moran, A. P., Athman, R., Memet, S., Huerre, M. R., Coyle, A. J., DiStefano, P. S., Sansonetti, P. J., Labigne, A., Bertin, J., Philpott, D. J., and Ferrero, R. L. (2004). Nod1 responds to peptidoglycan delivered by the Helicobacter pylori cag pathogenicity island. Nat. Immunol. 5, 1166-1174.

Willetts, N., and Wilkins, B. (1984). Processing of plasmid DNA during bacterial conjugation. Microbiol. Rev. 48, 24-41.

Wu, Z., Xu, L., Tu, Y., Chen, R., Yu, Y., Li, J., Tan, M., and Chen, H. (2011). The relationship between the symptoms of female gonococcal infections and serum progesterone level and the genotypes of Neisseria gonorrhoeae multiantigen sequence type (NG-MAST) in Wuhan, China. Eur. J. Clin. Microbiol. Infect. Dis. 30, 113-116.

Young, C., and Nester, E. W. (1988). Association of the VirD2 protein with the $5^{\prime}$ end of $\mathrm{T}$ strands in Agrobacterium tumefaciens. J. Bacteriol. 170, 3367-3374.

Zhu, J., Oger, P. M., Schrammeijer, B., Hooykaas, P. J. J., Farrand, S. K., and Winans, S. C. (2000). The bases of crown gall tumorigenesis. J. Bacteriol. 182, 3885-3895.

Zola, T. A., Strange, H. A., Domínguez, N. M., Dillard, J.P., and Cornelissen, C. N. (2010). Type IV secretion machinery promotes ton-independent intracellular survival of Neisseria gonorrhoeae within cervical epithelial cells. Infect. Immun. 78, 2429-2437.

Conflict of Interest Statement: The authors declare that the research was conducted in the absence of any commercial or financial relationships that could be construed as a potential conflict of interest.

Received: 01 February 2011; paper pending published: 14 February 2011; accepted: 21 March 2011; published online: 01 April 2011.

Citation: Ramsey ME, Woodhams KL and Dillard JP (2011) The gonococcal genetic island and type IV secretion in the pathogenic Neisseria. Front. Microbio. 2:61. doi: 10.3389/fmicb.2011.00061

This article was submitted to Frontiers in Cellular and Infection Microbiology, a specialty of Frontiers in Microbiology.

Copyright $\odot 2011$ Ramsey, Woodhams and Dillard. This is an open-access article subject to a non-exclusive license between the authors and Frontiers Media SA, which permits use, distribution and reproduction in other forums, provided the original authors and source are credited and other Frontiers conditions are complied with. 